\title{
The Explanatory Power of Adverse Relationship Experiences in Predicting Neuroticism
}

\author{
Kathryn Simms and Sara Bock \\ 700 Park Avenue, Norfolk State University, Norfolk, VA 23504, USA
}

\begin{abstract}
Positive correlations have been detected consistently between adverse relationship experiences (i.e., traumatic interpersonal events such as intimate partner violence) and a broad range of mental health disorders (e.g., depression). However, associations between adverse relationship experiences and personality have been under examined. Consequently, the purpose of this study was to evaluate the relationship between adverse relationship experiences and one facet of personality, neuroticism. Analyses consisted of Repeat Measures, Mixed Linear Modeling conducted on the National Longitudinal Study of Adolescent Health $(\mathrm{N}=3,726)$. Adverse relationship experiences explained $11 \%$ of the variability in neuroticism, whereas changes in self-reported neurotic symptomology over time explained $53 \%$ of the total variability in neuroticism. Adverse relationship experiences appeared to account for only a modest portion of self-reported neurotic symptomology and neurotic symptomology itself was relatively unstable.
\end{abstract}

Keywords: Neuroticism, Personality, State or Trait, Adverse Relationship Experiences

\section{INTRODUCTION}

Positive correlations have been detected consistently between adverse relationship experiences (i.e., traumatic interpersonal events such as domestic violence) and a broad range of mental health disorders (Adam et al., 2011; Kessler and Magee, 1993). However, associations between adverse relationship experiences and personality have been under examined. Consequently, the purpose of this study is to evaluate the relationship between adverse relationship experiences and one facet of personality, neuroticism. More specifically, this study seeks to advance extant literature by considering measures of adverse relationship experiences and neuroticism that are drawn from participants' comprehensive life experiences via the National Longitudinal Study of Adolescent Health (Add Health) (Harris, 2009). In doing so, this study has implications not only for establishing a more accurate understanding of the connection between adverse relationship experiences and neuroticism, but also for larger questions about the stability of neuroticism itself (Hampson and Goldberg, 2006; Terracciano et al., 2005).
More succinctly stated, this study evaluates the following research questions:

- Do adverse relationship experiences have explanatory power in accounting for variability in neurotic symptomology?

- Does neurotic symptomology itself vary over time?

As the summary of background literature in the next subsection indicates, formulating directional hypotheses about these research questions is difficult given scant and mixed findings in the literature (Costa et al., 2000; Engelhard et al., 2009). However, we tentatively hypothesize that consistent with untested conventional wisdom, adverse relationship experiences are highly correlated with neuroticism. Similarly, also consistent with untested conventional wisdom, we hypothesize that little variability exists in neurotic symptomology over time.

\subsection{Background}

Empirical connections have been detected consistently between adverse relationship experiences Corresponding Author: Kathryn Simms, 700 Park Avenue, Norfolk State University, Norfolk, VA 23504, USA 
and a broad range of mental health issues (e.g., depression (Adam et al., 2011; Kessler and Magee, 1993), conduct disorder (Tiet et al., 2001) and substance abuse (Bellis, 2002). Researchers (Kessler and Magee, 1993 ) initially studied the relevance of the character of adverse relationship experiences. In other words, what types of adverse relationship experience were correlated with poor mental health outcomes? Two themes have emerged for classifying adverse relationship experiences: (1) disrupted attachments in significant relationships (Adam et al., 2011; Kelley et al., 2003; Kessler and Magee, 1993; Kraaij et al., 2003; Mota et al., 2010; Repetti et al., 2002) and (2) physical violence and abuse (Adam et al., 2011; Ackard and Neumark-Sztainer, 2002; Kessler and Magee, 1993). However, specific terminologies for classifying types of adverse relationship experiences remained nearly as varied as the number of studies conducted on the topic.

Fortunately, the number of adverse relationship experiences, rather than the character of the adverse relationship experience, is the best predictor of poor mental outcomes (Adam et al., 2011; Anda et al., 2006; Chartier et al., 2010; Dube et al., 2003; Felitti et al., 1998). In other words, as the number--or the dosage--of adverse relationship experiences increases, the likelihood of mental health complications also increases.

Although the connection between dosage of adverse relationships and mental health disorders is well established, evidence is mixed about the connection between adverse relationship experiences and purportedly more stable, but problematic personality traits such as neuroticism. Some studies have detected positive associations between adverse relationship experiences and neuroticism (Costa et al., 2000; Whittington and Huppert, 1998), but other studies dispute this connection (Engelhard et al., 2009).

Inconsistencies in prior findings may have resulted in part from limitations in study designs. More specifically, many prior studies have had sampling constraints-such as being limited to high Socioeconomic Status (SES) samples (Costa et al., 2000)-or were otherwise group-specific (e.g., related to veterans (Engelhard et al., 2009). Additionally, no study appears to account for relatively ad hoc sampling of adverse relationship experiences and neuroticism. Instead, both adverse relationship experiences and neuroticism have been treated consistently as fixed effects. In reality, events and trait-related perceptions are generally measured based on narrow time periods (e.g., over the last two weeks, over the last year) that may not reflect individuals' more comprehensive dispositions over their lifetimes.

\section{MATERIALS AND METHODS}

\subsection{Participants}

Participants $(\mathrm{N}=3,726)$ in this study were main sample respondents to Waves I (1994-1995), II (1996) and IV (2007-2008) of the public-use version of the Add Health. The Add Health is considered to be largest longitudinal database of adolescents ever collected and provides a nationally representative sample of US 7-12 graders in 1994-1995 followed to adulthood. This database-collected by IRT International and administered at the University of North Carolina at Chapel Hill--was developed based on a multi-stage, stratified random sample. During the first stages of sampling, 80 high schools and 64 feeder junior and middle schools were selected. Subsequently, students in grades 7-12 were selected randomly from school rosters during Wave I for in-home interviews. These participants were also interviewed in their homes during other data waves. Note that data from Wave III (2001-2002) of the Add Health were not analyzed in this study because of inconsistencies in measures for Wave III compared to measures taken in Waves I, II and IV.

\subsection{Measures}

Congruent with the literature (Costa et al., 2000), neuroticism was measured as the mean of responses to the Anxious Personality Scale, the Center for Epidemiological Studies Depression Scale, temperamentalness, self-consciousness and negative effect. These and all other study measures were available in the Add Health database via participants' responses to in-home interviews. Dosages of adverse relationship experiences were assessed for each time period by counting the following occurrences: low parental support (i.e., lowest quartile); number of romantic relationships (i.e., highest quartile); intimate partner violence; and loss of a biological parent (Adam et al., 2011). Control variables included gender, race, age and SES (i.e., the higher of mother's or father's educational attainment).

\subsection{Data Analysis}

Following descriptive analysis, data were analyzed via a Repeat Measures, Mixed Linear Model (RMML). Although somewhat conceptually similar to Ordinary Least Squares (OLS), RMML differs from OLS in the following ways: 
- RMML corrects for serial correlation in error terms that results from longitudinal data analysis

- RMML provides fixed-point estimates, interpreted in a similar manner as unstandardized coefficients in OLS. RMML also yields estimates of random terms interpreted as the percentage of variability explained in the dependent variable

Five fixed effects were estimated, where the fixed effect with primary relevance to the study was the period of measurement, specified as a series of dummy variables (i.e., Period $1=$ Wave I, Period $2=$ Wave II and Period $3=$ Wave IV). Period 3 served as the contrast variable. Additional fixed effects included gender, race, age and SES. Random measures were repeated measures of neuroticism, dosage of adverse relationship experiences and--as is standard for RMML-participants. Treating participants as random accounted for participants being sampled rather than comprising the entire population under study.

\section{RESULTS}

\subsection{Descriptive Analysis}

For the full sample, means of neuroticism and dosage of adverse relationship experiences differed significantly across time (Table 1). Correlations between measures of neuroticism were strongest over the shorter interval between Period 1 to Period $2(0.59)$ and numerically weaker over longer time spans (i.e., 0.36 from Period 1 to Period 3; 0.37 for Period 2 to Period 3). The pattern of correlations was similar, but weaker for dosages of adverse relationship experiences (i.e., 0.26 for Period 1 to Period 2; 0.11 for both Period 1 to Period 3 and Period 2 to Period 3).

In supplemental analyses, we tested these findings for three subsamples of participants reporting the highest, middle and lowest ranges of neurotic symptomology during Period 1. The pattern of findings for these subsamples tended to be similar to those for the full sample-except that correlations were typically weaker.

\subsection{RMML}

Neuroticism was 0.33 higher for Period 1 compared to Period 3 and 0.30 higher for Period 2 compared to Period 3 indicating a decline in neuroticism over time (Table 2, fixed effects panel). Dosage of adverse relationship experiences was not determined to be a significant fixed effect during model development and consequently, was deleted from the final model in accordance with standard RMML modeling practices.

Table 1. Descriptive statistics for neuroticism and number of adverse relationship experiences

\begin{tabular}{llll}
\hline & Period 1 & Period 2 & Period 3 \\
\hline Neuroticism & & & 0 to 2.840 \\
Range & 0 to 3 & 0 to 2.950 & $0.775^{1,2}$ \\
Mean & $0.815^{2,3}$ & $0.800^{1,3}$ & 0.008 \\
Standard Error & 0.007 & 0.007 & \\
Correlations & 1 & & 1 \\
Period 1 & $0.587^{*}$ & $0.374^{*}$ & 1 \\
Period 2 & $0.360^{*}$ & & 0 to 4 \\
Period 3 & & 0 to 3 & $1.350^{1,2}$ \\
No. of adverse relationship exp. & & $0.870^{1,3}$ & 0.012 \\
Range & 0 to 4 & 0.01 & \\
Mean & $1.020^{2,3}$ & & 1 \\
Standard Error & 0.011 & 1 & \\
Correlations & & $0.114^{*}$ & \\
Period 1 & 1 & \\
Period 2 & $0.257^{*}$ & $0.108^{*}$ & \\
Period 3 & & \\
\end{tabular}

Note: Higher scores indicate greater neuroticism symptoms. Numerical superscripts indicate that means were significantly different between periods (e.g., $\mathrm{Mean}_{1}{ }^{2}$ indicates that the mean value for Period 1 differed significantly from the mean value for Period 2). Period 1 = wave I, 1994-1995; Period 2 = wave II, 1996; Period 3 = wave IV, 2007-2008; dosage of adverse relationship experience $=$ the total number of the following experiences: low parental support (i.e., lowest quartile), number of romantic relationships (i.e., highest quartile), intimate partner violence and death of a biological mother or father. ${ }^{*} p<0.05$ 
Kathryn Simms and Sara Bock / Current Research in Psychology 3 (2): 43-48, 2012

Table 2. Repeat measures, mixed linear model: Dosages of adverse relationship experiences and controls as predictors of neuroticism $(\mathrm{N}=3,726)$

\begin{tabular}{|c|c|c|c|}
\hline Fixed Effects & Coefficient & $\mathrm{t}$ & $\mathrm{p}$ \\
\hline Period 1 & 0.331 & 7.22 & 0.000 \\
\hline Period 2 & 0.297 & 6.92 & 0.000 \\
\hline Period 3 & - & - & - \\
\hline Male (45.8\%) & 0.136 & 11.58 & 0.000 \\
\hline Female $(54.2 \%)$ & - & - & - \\
\hline Black $(17.3 \%)$ & 0.044 & 2.76 & 0.006 \\
\hline Hispanic $(11.2 \%)$ & 0.063 & 3.23 & 0.001 \\
\hline \multicolumn{4}{|l|}{ Asian/Pacific } \\
\hline Islander $(2.7 \%)$ & 0.112 & 3.08 & 0.002 \\
\hline Native American (.5\%) & 0.095 & 1.19 & 0.234 \\
\hline Multi-racial $(4.7 \%)$ & 0.078 & 2.8 & 0.005 \\
\hline \multicolumn{4}{|l|}{ Non-Hispanic } \\
\hline White (63.6\%) & - & - & - \\
\hline \multicolumn{4}{|c|}{ Age (Period 1: 11 to 21 , Period 2: 11 to 21 ; Period 3: 24 to 33 ) } \\
\hline & 0.022 & 6.38 & 0.000 \\
\hline \multicolumn{4}{|l|}{ Parental education } \\
\hline Less than high school $(4.9 \%)$ & 0.019 & 0.65 & 0.514 \\
\hline At least college $(35.1 \%)$ & -0.096 & -7.65 & 0.000 \\
\hline \multicolumn{4}{|l|}{ High School $(60 \%)$} \\
\hline & - & - & - \\
\hline Intercept & 0.087 & 0.87 & 0.383 \\
\hline Random Effects & Estimate & Wald Z & $\mathrm{p}$ \\
\hline Repeat measures (neuroticism) & 0.109 & 46.46 & 0.000 \\
\hline Intercept (participant) & 0.075 & 22.76 & 0.000 \\
\hline Dosage & 0.023 & 7.26 & 0.000 \\
\hline
\end{tabular}

Note: Coefficients are unstandardized coefficients. Higher scores indicate greater neuroticism symptoms. Period $1=$ wave I, 19941995; Period 2 = wave II, 1996; Period 3 = wave IV, 2007-2008. Parental education = higher of mother's or father's education level; Dosage (of adverse relationship experience) = the total number of the following experiences: low parental support (i.e., lowest quartile), number of romantic relationships (i.e., highest quartile), intimate partner violence and death of a biological mother or father

However, dosage of adverse relationship experiences explained $11 \%$ of the variability in neurotic symptomology over time (Table 2, random effects panel: $0.023 /(0.109+0.075+0.023))$. This explanatory power was weaker than the $53 \%$ of variability in neurotic symptomology attributed to repeat measurement (Table $\mathbf{2}$, random effects panel: $0.109 /(0.109+0.075+0.023))$.

\section{DISCUSSION}

The relationship between adverse relationship experiences and mental health disorders is well established in the literature (Adam et al., 2011; Kessler and Magee, 1993). By contrast, the connection between adverse relationship experiences and purportedly more stable traits, such as personality, has been understudied. Additionally, the conclusions of the relatively few extant studies available are limited. These limitations derive from sampling constraints (Costa et al., 2000;
Engelhard et al., 2009) and methodological concerns (e.g., personality traits are measured via a single "snapshot" approach and accordingly may not reflect participants' lifetime dispositions).

This study contributes to the literature by assessing the relationship between one facet of personalityneuroticism-and adverse relationship experiences. It compensates for prior methodological constraints by relying on a nationally representative sample of U.S. participants and properly treats neurotic symptomology as a random effect. In terms of the main research questions of this study, dosages of adverse relationship experiences were associated with only a modest portion (11\%) neurotic symptomology. Supplemental analysis indicated that participants with higher levels of initial neurotic symptomology did not experience atypical patterns of adverse relationship experiences relative to other participants. The finding that over half of the variability in neuroticism was due to changes in reported 
neurotic symptomology also suggests the connection between adverse relationship experiences and neuroticism was relatively tenuous.

Any study should be interpreted in consideration of limitations in its design. In particular, measures in this study were self-reported. Additionally, analyses were based on correlations, not causation. Future research should consider the connection between adverse relationship experiences and other personality traits as well as explore the connection between personality traits and adverse relationship experiences in multinational samples.

\section{CONCLUSION}

This study has important implications for clinical practice. In particular, a well-established concern among practicing psychologists and other clinicians has been that adverse relationship experiences are merely a byproduct of neurotic symptomology. Consequently, the long-term efficacy of therapy designed to remedy these relationship patterns among neurotic patients has generally been questioned. Furthermore, there has been wide concern that treating neurotic symptomology itself might be dubious, given that personality is traditionally viewed as largely stable.

However, this study suggests that neuroticism is not as likely to be as persistent as previously hypothesized, nor are adverse relationship experiences highly predictive of neuroticism. Consequently, patients with neurotic symptomology might benefit from interventions focused on both resolving current adverse relationship experiences (if these relationships are present) and neurotic symptomology itself. Clinicians may have less need to be concerned that adverse relationship experiences and even neurotic symptoms themselves will be cyclical.

\section{ACKNOWLEDGEMENT}

This study was presented at the Virginia Psychological Association (VPA) Spring Convention on April 26, 2012 in Norfolk, Virginia, where it received the William Applegate Award for the Best Graduate Paper in an Applied Area. No author of this study received a grant specific to this study. However, when this study was conducted both authors received their salaries from Title III \#2140053: Strengthening Research Capacity in the College of Liberal Arts (9-2010 to 9-2012). This grant was designed to encourage any type of research in the College of Liberal Arts at (deleted for peer review).

\section{REFERENCES}

Ackard, D.M. and D. Neumark-Sztainer, 2002. Date violence and date rape among adolescents: Associations with disordered eating behaviors and psychological health. Child Abuse Neglect, 26: 455473. DOI: $10.1016 / \mathrm{S} 0145-2134(02) 00322-8$

Adam, E.K., L. Chyu, L.T. Hoyt, L.D. Doane and J. Bolisyoly et al., 2011. Adverse adolescent relationship histories and young adult health: Cumulative effects of loneliness, low parental support, relationship instability, intimate partner violence and loss. J. Adolescent Health, 49: 278286. DOI: 10.1016/j.jadohealth.2010.12.012

Anda, R.F., V.J. Felitti, D.J. Bremner, J.D. Walker and C. Whitfield et al., 2006. The enduring effects of abuse and related adverse experiences in childhood. Eur. Arch. Psychiatry Clin. Neurosci., 256: 174-186. DOI: $10.1007 / \mathrm{s} 00406-005-0624-4$

Bellis, M.D.D., 2002. Developmental traumatology: A contributory mechanism for alcohol and substance use disorders. Psychoneuroendocrinology, 27: 155 170. DOI: 10.1016/S0306-4530(01)00042-7

Chartier, M.J., J.R.Walker and B. Naimark, 2010. Separate and cumulative effects of adverse childhood experiences in predicting adult health and health care utilization. Child Abuse Neglect, 34: 454-464. DOI: 10.1016/j.chiabu.2009.09.020

Costa, P.T., J.H. Herbst, R.R. McCrae and I.C. Siegler, 2000. Personality at midlife: Stability, intrinsic maturation and response to life events. Assessment, 7: 365-378. DOI: 10.1177/107319110000700405

Dube, S.R., V.J. Felitti, M. Dong, W.H. Giles and R.F. Anda, 2003. The impact of adverse childhood experiences on health problems: Evidence from four birth cohorts dating back to 1900 . Preventive Med., 37: 268-277. DOI: 10.1016/S0091-7435(03)00123-3

Engelhard, I.M., M.A.V.D. Hout and M.J. Lommen, 2009. Individuals high in neuroticism are not more reactive to adverse events. Personality Individual Differ., 47: 697-700. DOI: 10.1016/j.paid.2009.05.031

Felitti, V.J., R.F. Anda, D. Nordenberg, D.F. Williamson and A.M. Spitz et al., 1998. Relationship of childhood abuse and household dysfunction to many of the leading causes of death in adults: The Adverse Childhood Experiences (ACE) study. Am. J. Preventive Med., 14: 245-258. DOI: 10.1016/S07493797(98)00017-8 
Hampson, S.E. and L.R. Goldberg, 2006. A first large cohort study of personality trait stability over the 40 years between elementary school and midlife. J. Personality Soc. Psychol., 91: 763-779. DOI: 10.1037/0022-3514.91.4.763

Harris, K.M., 2009. The national longitudinal study of adolescent health (add health), Waves I and II, 1994-1996; Wave IV, 2007-2009 [Data file and codebook]. University of North Carolina, Chapel Hill, NC.

Kelley, S.S., E.A. Borawski, S.A. Flocke and K.J. Keen, 2003. The role of sequential and concurrent sexual relationships in the risk of sexually transmitted diseases among adolescents. J. Adolescent Health, 32: 296-305. DOI: 10.1016/S1054-139X(02)007103

Kessler, R.C. and W.J. Magee, 1993. Childhood adversities and adult depression: Basic patterns of association in a US national survey. Psychol. Med., 23: 679-690. DOI: 10.1017/S0033291700025460

Kraaij, V., N. Garnefski, E.J.D. Wilde, A. Dijkstra and W. Gebhardt et al., 2003. Negative life events and depressive symptoms in late adolescence: Bonding and cognitive coping as vulnerability factors? J. Youth Adolescence, 32: 185-193. DOI: 10.1023/A:1022543419747
Mota, N.P., B.J. Cox, L.Y. Katz and J. Sareen, 2010. Relationship between mental disorders/suicidality and three sexual Behaviors: Results from the National comorbidity survey replication. Arch. Sexual Behav., 39: 724-734. DOI: 10.1007/s10508008-9463-5

Repetti, R.L., S.E. Taylor and T.E. Seeman, 2002. Risky families: Family social environments and the mental and physical health of offspring. Psychol. Bull., 128: 330-366. DOI: 10.1037/0033-2909.128.2.330

Terracciano, A., R.R. McCrae, L.J. Brant and P.T. Costa, 2005. Hierarchical linear modeling analyses of the NEO-PI-R scales in the Baltimore longitudinal study of aging. Psychol. Aging, 20: 493-506. DOI: 10.1037/0882-7974.20.3.493

Tiet, Q.Q., H.R. Bird, C.W. Hoven, R. Moore and P. Wu et al., 2001. Relationship between specific adverse life events and psychiatric disorders. J. Abnormal Child Psychol., 29: 153-164. DOI: 10.1023/A:1005288130494

Whittington, J.E. and F.A. Huppert, 1998. Neuroticism, psychiatric symptoms and life events. Personality Individual Differ., 24: 97-107. DOI: 10.1016/S01918869(97)00151-7 